\title{
From Activity Recognition to Intention Recognition for Assisted Living within Smart Homes
}

\author{
J. Rafferty, IEEE Member, C. Nugent, IEEE Member, J. Liu, IEEE Member, and L. Chen, IEEE \\ Member
}

\begin{abstract}
The global population is aging; projections show that by 2050 , over $20 \%$ of the population will be aged over 64 . This will lead to an increase in aging related illness, a decrease in informal support, and ultimately issues with providing care for these individuals. Assistive Smart Homes provide a promising solution to some of these issues. Nevertheless, they currently have issues hindering their adoption. To help address some of these issues, this study introduces a novel approach to implementing assistive Smart Homes. The devised approach is based upon an Intention Recognition mechanism incorporated into an intelligent agent architecture. This approach is detailed and evaluated. Evaluation was performed across three scenarios. Scenario 1 involved a web interface, focusing on testing the Intention Recognition mechanism. Scenarios 2 and 3 involved retrofitting a home with sensors and providing assistance with activities over a period of 3 months. The average accuracy for these three scenarios was $100 \%$, $64.4 \%$, and $83.3 \%$, respectively. Future will extend and further evaluate this approach by implementing advanced sensor-filtering rules and evaluating more complex activities.
\end{abstract}

Index Terms - Intention Recognition, Ambient Assisted Living, Smart Homes, Intelligent Agents, Goal Recognition, Activity Recognition

\section{INTRODUCTION}

$\mathrm{T}$ he worldwide population is ageing and is resulting in an uneven demographic composition [1], [2]. This is expected to reach a situation where by 2050 over $20 \%$ of the population will be aged over 64 [1], [2]. This growth in the aging population is expected to produce an increase in agerelated illness which, in turn, will place additional burdens on healthcare provision [2]. In addition, the amount of informal support available will decrease due to a reduction in the global Potential Support Ratio (PSR). The PSR is the ratio of people that comprise the working age (15-64) to those older than 64 [1]. The PSR is expected to continue on a downward trend reaching a low of $4: 1$ by 2050 . The PSR was previously 12:1 in 1950 and more recently 9:1 in 2009 [1].

Ambient Assisted Living (AAL) has been widely viewed as a promising approach to address some of the problems associated with supporting the ageing population [3], [4].

This article was submitted for review on the $27^{\text {th }}$ December 2015. This study was supported in part by the Northern Ireland Department of Education and Learning through its $\mathrm{PhD}$ scholarship programme.

J. Rafferty, C. Nugent, and J. Liu are with the School of Computing and Mathematics, Ulster University, Jordanstown, Newtownabbey, UK, BT37 0QB (e-mail: j.rafferty@ulster.ac.uk, cd.nugent@ulster.ac.uk, j.liu@ulster.ac.uk).

L. Chen is with the School of Computer Science and Informatics, De Montfort University, The Gateway, Leicester, LE1 9BH, (e-mail: liming.chen@dmu.ac.uk)
Within the context of AAL, technology-based solutions are used to support independent living and subsequently alleviate a portion of the problems associated with ageing. Such an approach offers the potential of enhancing the quality of life of older people. The notion of Smart Homes (SH), namely residential environments augmented with sensor technology and assistive services have emerged as a dominant realization of the AAL approach.

Typically, SHs operate in a 'bottom-up' process, as presented in Fig. 1. In this paradigm, sensors monitor an inhabitant's activities/environment. Data from these sensors are collected and processed to identify Activities of Daily Living (ADL), such as washing or preparing a meal. By monitoring ADLs in this manner, it is possible to detect difficulties in task completion subsequently allowing assistance to be offered through the SH services [3]-[6]. As such, SHs allow older people to live longer independently, with a better quality of life, in their own homes.

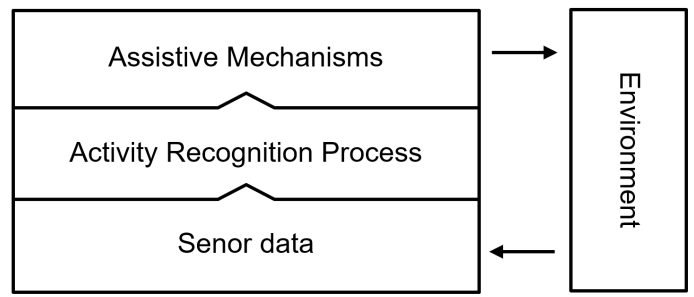

Fig. 1. An illustration of the 'bottom-up' process used to realize smart homes.

The bottom-up approach, although functional, has issues that stem from its sensor centric nature. This study incorporates a novel approach to realizing a SH platform that addresses the specific issues of privacy, reusability, scalability and applicability. This novel approach is achieved through a paradigm shift incorporating Intention Recognition (IR) in conjunction with an Intelligent Agent architecture.

The remainder of this article is organized as follows. Section II presents related studies; Section III presents the overall architecture of this Intelligent Agent-based SH and details the novel IR approach core to realizing this study. Section IV describes testing and evaluation experiments, and discusses the performance of this approach. Section V elaborates future research, followed by Conclusions in Section VI.

\section{RELATED STUDIES}

The related studies for this research broadly fall into three categories: SHs enabling AAL, Intelligent Agent architectures, and IR techniques. 


\section{A. Smart Homes enabling Ambient Assisted Living}

A wide range of works exist related to Smart Home-enabled AAL, which span many specific approaches and design goals. At the core of these systems is sensor-based activity recognition to provide assistance with, or monitoring of, Activities of Daily Living (ADLs).

\section{A.1 Activity Recognition Approaches Within Smart Homes}

Broadly speaking, there are two main approaches to activity recognition in current $\mathrm{SH}$ work, namely data driven and knowledge driven.

Data driven approaches use statistical and probabilistic methods to learn activity models from datasets. In these approaches, datasets are a collection of sensor activations generated from a SH. These datasets are then used to train activity models that map the relationship between events and activities. The learnt activity models are then used to perform future recognition of the events recorded within the $\mathrm{SH}$. The learning mechanisms are usually based on two types of data mining and machine learning methods, namely, generative and discriminative depending on the modeling strategy employed.

Generative approaches, such as those used in [7]-[11], attempt to produce a description of occurrences in a dataset by fully mapping the relationship of sensor events and activities. These mappings identify the most likely activities that would occur given a set of observations. This classification of observations from a dataset is achieved using probabilistic classification techniques such as Hidden Markov Models or naïve Bayes classifiers. Generative approaches suffer from the requirement of having a sufficient amount of data being available to produce the complete set of probabilistic representations to provide good functionality. Discriminative approaches, such as those used in [12]-[16], can produce results using a less exhaustive dataset compared to generative approaches. These approaches focus on matching input states (sensor data) to activity labels (classification). This approach may use techniques such as Artificial Neural Networks.

The general advantages of data driven approaches are that they allow the modeling of uncertainty and temporal parameters. Their disadvantages include the need to have a suitably large dataset to learn from. Additionally, the reusability of these activity models is limited to the environment and scenarios that have produced the dataset.

Knowledge-driven approaches to activity recognition use domain knowledge and a priori heuristics as the basis to create activity models. Domain knowledge is an intuitive record, learned though human experience and does not require a large number of formally recorded sensor and activity records from which to generate activity models. Knowledge driven approaches are generally logical or ontological in nature.

Logical based approaches, such as those in [17], [18], encode representations of ADLs into logical structures using knowledge representation formalisms. These logical structures are combined with knowledge-based inference to support activity recognition. Across the various logical approaches, the knowledge formalisms used for activity modeling and recognition may vary. The overall process is common and is described as follows. Domain knowledge is gathered to define activities and their performance. Approaches and formalisms based on knowledge modeling are subsequently used to create logical representations of the activities, for example, encoding plans into a lattice structure [18]. Reasoning mechanisms are applied to map changes in state with the aim of determining what, if any, activities are occurring. Sensor and activity ontologies have been used in [19]-[24] as the basis for knowledge-driven activity recognition and AAL applications.

Ontological modeling [25] allows explicit representation of a domain concept. This is achieved by structuring elements into a hierarchy of concepts and classes. These classes and concepts can have properties, relationships, and restrictions. The flexibility of ontologies has been leveraged to allow greater reuse of activity representations [19]. This implementation overcomes the flexibility issues that traditional logical approaches have encountered from their use of rigid activity representations. In this approach, common activity representations are used to provide generic representation of ADLs. When modeling the performance of an activity by an inhabitant, a relevant common representation is used to produce a personalized representation of a specific ADL.

Logic-based approaches do not require a dataset to provide training for the activity recognition mechanisms. This frees them from exclusive use with the environment and scenario that produced the dataset [3]-[5], [19], [26]. Additionally, these approaches have clear operation as the mechanisms of activity recognition and encodings of ADL sets are explicitly defined. These approaches have some negative aspects, namely, difficulty in representing uncertainty and the relatively rigid representations of ADL sets providing limited personalization.

Ontological approaches add to the benefits of logical approaches through the addition of flexible models and allowing greater reusability inherent to ontological structures. Disadvantages include weakness in handling uncertainty and modeling, as with other current logical approaches. A plethora of studies relating to activity recognition and SHs currently exists with existing literature reviews [3]-[6], [27] providing further coverage of a large number of these studies.

\section{A. 2 Problems Faced by Activity Recognition in Smart Homes}

In summary, current approaches to activity recognition in SHs have a number of issues, which are:

1) inhabitant privacy is potentially violated by recording activities that are then used as the basis for providing assistive services [3]-[6].

2) activity models may be expressed through complex languages, requiring specialist knowledge beyond those related to the activities to be modeled.

3 ) to perform efficiently, SHs require a large number of sensors in the environment, which is not feasible for widespread use because of scalability issues related to retrofitting a large number of homes with an appropriate suite of sensors. This retrofitting process presents a substantial financial cost in addition to disturbance to inhabitants within their own homes. These sensor installations also require maintenance representing a potential further cost and disturbance [3]-[6].

4) current SHs using data driven or logical driven approaches cannot handle variation in activity performance in a satisfactory manner [3]-[6]. 
5) reusability of some of these bottom-up $\mathrm{SHs}$ can be reduced as they rely on a record of events that occur only within their environment [3]-[6].

To address these issues, a move from a sensor centric approach to a 'top-down' goal-driven approach has been proposed [28]. This offers a solution that can offer additional flexibility while simultaneously requiring fewer sensors.

In a goal-driven approach, an inhabitant's goals are the focus of the assistive system. This is in contrast to the processing of dense sensor recordings. By combining a goal/IR system with an action planning mechanism, an assistive Intelligent Agentbased system can be produced. This goal-driven approach will allow flexible and proactive assessment of an intended inhabitant goal, thus facilitating assistance provision. This assistance will be in the form of dynamically generated, videobased instruction.

Previous studies have been published covering the dynamic generation of video-based instruction for inhabitant goals [29], [30] and is the overall approach devised in this study [28].

\section{B. Intelligent Agent Architectures}

Intelligent Agents are software entities that model the environment within which they are situated and affect changes within it to achieve a state they desire [31], [32]. Intelligent Agents can be realized in many different ways, as dictated by the properties of agency that they wish to possess, such as:

1) autonomy - operation without intervention.

2) social capability - coexistence and collaboration with other Intelligent Agents and/or humans.

3) reactivity - an Intelligent Agent can react to changes in its world model.

4) proactiveness - Intelligent Agents can act toward achieving goals that they hold and not simply respond to changes in their environment.

Realization of an Intelligent Agent is directly related to how these key properties are incorporated, knowledge is represented, and reasoning is performed [31], [33], [34].

One of the most prevalent Intelligent Agent implementation methods is that of Beliefs, Desires and Intentions (BDI) [32]. BDI is based on modeling Beliefs, Desires, and Intentions from an abstraction of human cognition [35]. In the BDI model, Beliefs represent the states of the world, as an agent perceives it (corresponding to knowledge). Desires provide some motivation for an agent's action (corresponding to potential goals). Finally, Intentions are desires that an agent has committed to achieving through performance of related actions (corresponding to a plan). BDI is a concept that has been realized through many different architectural implementations, which vary depending on the aim of the designer of the Intelligent Agent [31], [34], [36]-[39].

Contemporary use of Intelligent Agents within a SH operate in an agent-centric manner, wherein an agent models and works toward their own goals, such as climate control. These goals are, in essence, a flexible form of activity model employed by these Intelligent Agents.

Much like inhabitants of a SH, Intelligent Agents work toward their goals by perceiving their environment, reasoning, and then acting. Similarly, combined with how BDI is based on human cognition models [31], [36], BDI Intelligent Agents may be used to model and represent the goals and requirements of inhabitants. Specifically, the operation and properties of an Intelligent Agent can correspond to behavioral and cognitive elements of a SH inhabitant.

Beliefs are analogous to the understanding that an inhabitant has about their environment, such as active appliances in a kitchen. Desires are akin to the goals that an inhabitant would have, such as making a drink. Finally, Intentions are similar to the actions that an inhabitant would perform to achieve desires, such as reaching for a cup. BDI agents can provide an assistive function within a $\mathrm{SH}$ by modeling inhabitant goals in the context of beliefs about the smart environment and realizing intentions through inhabitant guidance. Such an approach would use Intelligent Agents in a novel manner, introducing an element of goal surrogacy to providing assistance with ADLs [3]-[5], [27], [40]-[42] and would provide the following advantages over current approaches:

1) flexibly modeling inhabitant activity through inhabitant goal models, addressing inflexible activity models.

2) providing reusable, extensible, and sharable inhabitant goal models by using semantic web technologies, and addressing some issues with scalability.

3) performing activity recognition functions through intended goal recognition utilizing a reduced set of sensors, addressing both the need for expensive sensor suites and some scalability problems.

4) providing dynamic assistance in the form of prompting through agent-based planning from actions in inhabitant goals.

5) identifying and assisting multiple inhabitants through assignment of an agent per inhabitant, addressing multiple occupants and some scalability issues.

Core to realization of such an approach would be a process of recognizing the intention of a $\mathrm{SH}$ inhabitant. An overview of current IR techniques is presented in Section $\mathrm{C}$, with a focus placed on those used in AAL/SHs.

\section{C.Intention Recognition Techniques}

Intention Recognition involves predicting the most likely intended goal/action of persons, entity, or agent. This is achieved by observing their actions and reasoning about them. These observations are considered by a predictive reasoning system in conjunction with a library of relevant goals [43], [44].

Three classes of IR operation have been identified [43]-[45], these are intended, keyhole, and adversarial.

Intended IR is where an observed entity is aware of this observation and openly provides signals to allow other entities to ascertain their intention. An example of intended IR is communicating instructions to make a phone call to a contact through to a personal digital assistant on a smartphone. This will provide full observability into actions.

Keyhole IR is where an entity is being observed and does not openly or knowingly intend for their actions to be observed. An example of this is an inhabitant's day to day activities, which are being observed by an assistive agent.

Adversarial IR is where an entity does not wish for their intentions to be known, and act in a misleading or actively concealed manner. An example of this is a malicious actor engaging in criminal acts. 
To realize IR systems, three components are typically combined, these are:

1) a library of goals that may be achieved and recognized.

2) a record of observed actions, as performed by the target of the IR mechanism.

3) knowledge about goal achievement through actions, typically incorporating a theory of action.

Currently, IR techniques are used in a number of domains, including anti-terrorism, cyber-security, and military planning, in addition to limited use in SHs and AAL [46], [47]. The small amount of research into IR for SHs and AAL have limited utility. Limitations stem from the activity models used and their design goals. Specifically, these studies do not model inhabitant goals in a manner that can provide assistance appropriately, rely on goal models with limited reusability, or are theoretical in nature [46], [48], [49], [50], [51].

Computational State Space Models (CSSM) represent a promising method for IR in the context of AAL [51], [52]. These studies have shown promise, however, have a number of detractors related to scalability. Specifically, modeling activities are an intensive process, and the authors state it could take one week to model a specific task. Additionally, through using computational action languages, expertise outside of the area of domain knowledge is likely required, specifically with a computer science background.

Agent-based IR within the domain of AAL would require a goal model incorporating both declarative and procedural aspects. Declarative aspects are essentially metadata about a goal that an inhabitant is pursing; this allows deliberation about the pursuit of inhabitant goals. Procedural aspects are stepwise instructions detailing how inhabitant goals may be achieved.

Such a novel goal model has been produced by the authors in previous studies [28]. This goal model is the core of the novel IR approach developed for use within this agent-based system. Both the novel IR system and agent-based system are detailed in Section III, followed by an evaluation in Section IV.

\section{THE AGENT-BASED APPROACH TO INTENTION RECOGNITION}

In the devised agent-based system, the BDI paradigm has been adopted. Specifically, beliefs represent the agent's perception of the world, desires are represented by a library of inhabitant goals, and intentions are goals an inhabitant is pursuing as detected through an IR system. Beliefs and desires are modeled through the use of ontologies, allowing greater reusability of these models over traditional approaches; in addition, this allows these concepts to be linked through the semantic web. Further details of this approach are presented in the following Sections.

\section{A. Desires}

In this goal-driven agent, ADLs, and constituent tasks of ADLs, are represented by a library of goals. These goals consist of atomic actions, activation conditions, and metadata. Atomic actions are the steps required to achieve specific goals/tasks. Activation conditions are states repressing specific triggers for particular goal conditions. Goals may be in a number of states which are suspended, eligible, and in need of assistance. Metadata assists with modeling of goals in a human-friendly form and enables production of goals with component subgoals. These goals and actions support preconditions to provide an indication of the order that such actions need to be completed in, or any dependent actions. Fig. 2 shows the ontological goal model to be used in this approach, which is presented as a hierarchy of concepts, and is detailed further in Table I.

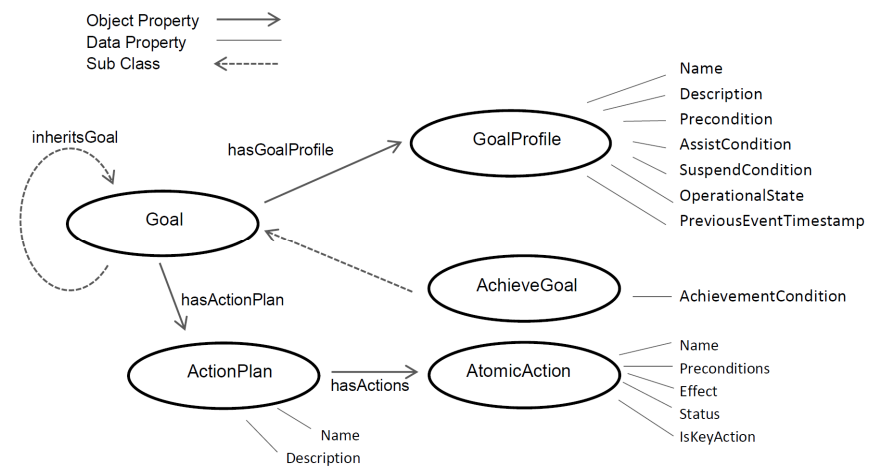

Fig. 2. The classes, object properties and data properties of the goal ontology presented as a hierarchy of concepts.

TABLE I

THE PROPERTIES OF THE BASE GOAL ONTOLOGY.

\begin{tabular}{|c|c|}
\hline Property & Description \\
\hline Name & $\begin{array}{l}\text { A name for the GoalProfile, ActionPlan or } \\
\text { AtomicAction }\end{array}$ \\
\hline Description & $\begin{array}{l}\text { A description of the GoalProfile, ActionPlan or } \\
\text { AtomicAction }\end{array}$ \\
\hline OperationalState & $\begin{array}{l}\text { The current state of the goal. Typically, this will be } \\
\text { an enumeration of } 1 \text { of } 4 \text { eligible states; : Inactive" } \\
\mid \text { "Active" | "Assist" | "Suspended"\}. }\end{array}$ \\
\hline SuspsendCondition & $\begin{array}{l}\text { This represents conditions where a goal is } \\
\text { considered to be suspended, such as } \\
\text { \{notIntendedGoal : true\} }\end{array}$ \\
\hline AssistCondition & $\begin{array}{l}\text { This represents a condition where a goal is in need } \\
\text { of assistance, such as }\{\text { thresholdLastAction: } 500\}\end{array}$ \\
\hline Precondition & $\begin{array}{l}\text { Optional. Needed for an AtomicAction or goal to } \\
\text { become optional, such as \{goalCompleted: } \\
\text { anotherGoal\} }\end{array}$ \\
\hline $\begin{array}{l}\text { PreviousEventTimestam } \\
p\end{array}$ & $\begin{array}{l}\text { Time stamp of a previous goal action as represented } \\
\text { by Unix time, such as } 511582480\end{array}$ \\
\hline AchievementCondition & $\begin{array}{l}\text { This condition under which a goal is considered to } \\
\text { be achieved. All the actions to complete the goal } \\
\text { have been performed, such as } \\
\text { \{completeActionCount : } 3 \text { \} }\end{array}$ \\
\hline Effect & $\begin{array}{l}\text { Optional. An effect to be expressed when this action } \\
\text { is complete. \{assertGoal: anotherGoal\} }\end{array}$ \\
\hline Action status & $\begin{array}{l}\text { A Boolean flag showing if this action has been } \\
\text { completed or not, True or False. }\end{array}$ \\
\hline IsKeyAction & A Boolean flag to indicate if this is a key action. \\
\hline
\end{tabular}

Additionally, goals can both have subgoals and be subgoals by use of the inheritsGoal property. As such, in contrast with ADLs, goals may represent small tasks that are achieved and contribute to a goal, which is an ADL. This allows a hierarchy of goals to form from a number of reusable modeled goals, which may contain actions, as presented in Fig. 3.

The hierarchy presented in Fig. 3 presents the flexibility of this goal model. In this instance, 13 atomic actions are used to flexibly model 16 potential user goals. These specific goals are shown in Table II. 


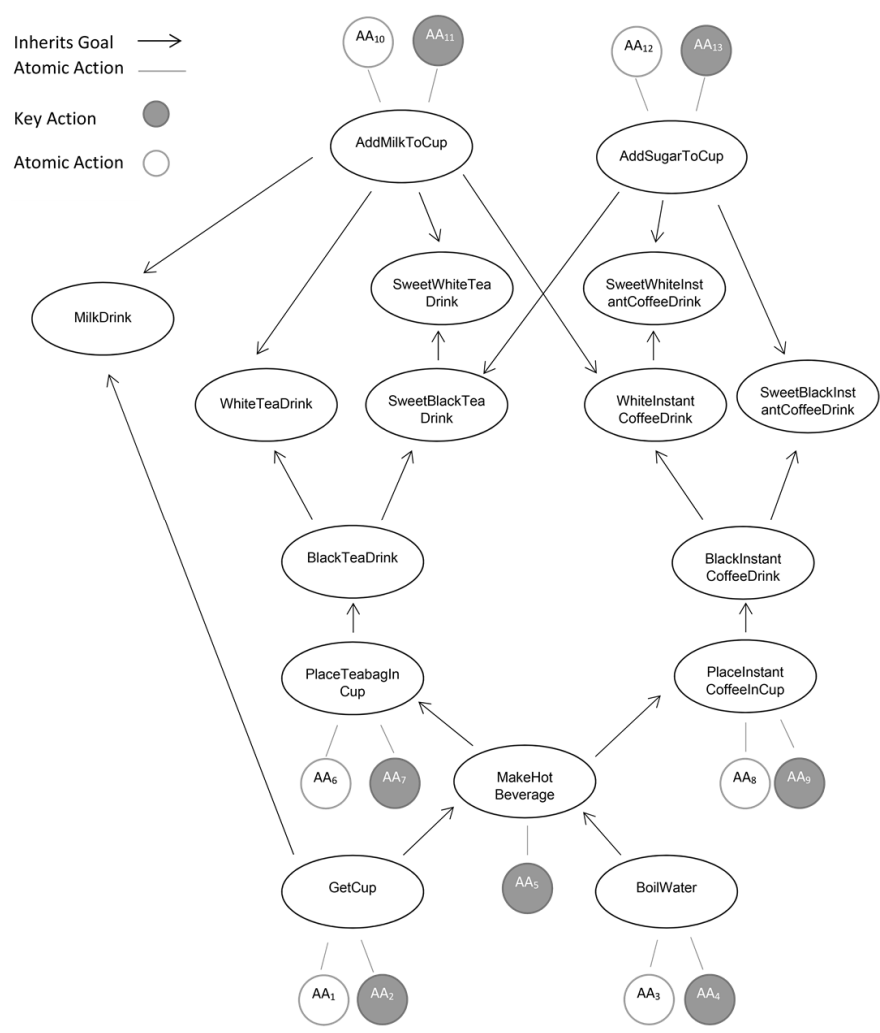

Fig. 3. An example of a goal hierarchy modeled through goal inheritance. Key atomic actions are shaded.

TABLE II

AN EXAMPLE OF COMBINING GOALS WITH AND WITHOUT ATOMIC ACTIONS, PRODUCING THE HIERARCHY SHOWN IN FIGURE 3.

\begin{tabular}{|c|c|c|}
\hline Goal name & Atomic actions & Inherited Goals \\
\hline SweetWhiteInstant & & AddSugarToCup \\
\hline CoffeeDrink & & WhiteInstantCoffeeDrink \\
\hline WhiteInstantCoffeeDrink & & BlackInstantCoffeeDrink \\
\hline $\begin{array}{c}\text { SweetBlackInstantCoffee } \\
\text { Drink }\end{array}$ & & AddMilkToCup \\
\hline BlackInstantCoffeeDrink & & AddSugarToCup \\
\hline SweetWhiteTeaDrink & & BlackInstantCoffeeDrink \\
\hline WhiteTeaDrink & & MakeHotBeverage \\
\hline SweetBlackTeaDrink & & PlaceInstantCoffeeInCup \\
\hline BlackTeaDrink & & AddSugarToCup \\
\hline MilkDrink & & WhiteTeaDrink \\
\hline AddSugarToCup & $\begin{array}{c}\text { AA12 - } \\
\text { ObtainSugarVessel }\end{array}$ & BlackTeaDrink \\
\hline AddMilkToCup & $\begin{array}{c}\text { AA13 - } \\
\text { AccessContentsOfSugar } \\
\text { Vessel }\end{array}$ & AddMilkToCup \\
\hline MakeHotBeverage & $\begin{array}{c}\text { AA11 - } \\
\text { ObtainMilkVessel }\end{array}$ & AddSugarToCup \\
\hline PlaceInstantCoffeeInCup & AA10 - PourMilk & BlackTeaDrink \\
\hline PlaceTeabagInCup & AA5 - PourBoiledWater & MakeHotBeverage \\
\hline \multirow[t]{2}{*}{ GetCup } & $\begin{array}{c}\text { AA8 - } \\
\text { ObtainCoffeeVessel }\end{array}$ & PlaceTeabagInCup \\
\hline & $\begin{array}{c}\text { AA9 - } \\
\text { AccessContentsOfCoffee } \\
\text { Vessel }\end{array}$ & GetCup \\
\hline BoilWater & $\begin{array}{c}\text { AA6 - } \\
\text { ObtainTeabagVessel }\end{array}$ & \\
\hline $\begin{array}{l}\text { SweetWhiteInstant } \\
\text { CoffeeDrink }\end{array}$ & $\begin{array}{c}\text { AA7 - } \\
\text { AccessContentsOf } \\
\text { TeabagVessel }\end{array}$ & \\
\hline
\end{tabular}

\section{B. Beliefs}

In this goal-driven agent, beliefs are a record of sensor activations. These sensor activations are in turn a representation of actions being performed by an inhabitant.

These sensors are modeled in a belief ontology, which additionally models the association with the atomic actions they represent. The structure for this belief ontology is shown in Fig. 4.

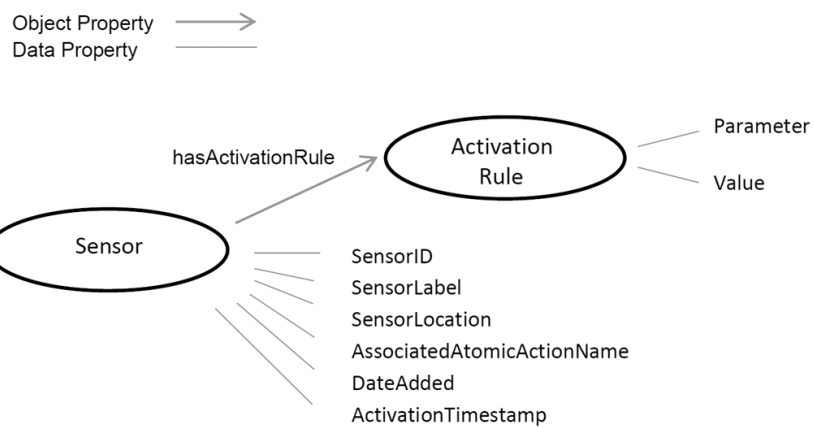

Fig. 4. The classes, object properties and data properties of the belief ontology.

In this belief ontology, sensors are given profiles that record relevant metadata including those associated to atomic actions via the AssociatedAtomicActionName data property. These AssoiciatedAtomicActionName properties are linked to the goal ontology via SPARQL Protocol and RDF Query Language (SPARQL) rules. SPARQL rules can be used to query linked semantic data across modeled concepts. These SPARQL rules are shown in (1), below.

SELECT DISTINCT ?sensorBlankNode ?sensorID

?SensorLabel ?sensorLocation

?activationTimestamp WHERE \{ OPTIONAL

\{?sensorBlankNode ug:SensorID ?sensorID

.OPTIONAL \{?sensorBlankNode ug:SensorLabel

?SensorLabel\} .OPTIONAL \{?sensorBlankNode

ug:SensorLocation ?sensorLocation .

?sensorBlankNode ug:ActivationTimestamp

?activationTimestamp \}

SELECT ?activationRule WHERE \{OPTIONAL

\{?sensorBlankNode ug:hasActivationRule

?activationRule \}\}

\section{SELECT ?associatedAtomicActionName WHERE \{ OPTIONAL \{?sensorBlankNode ug:AssociatedAtomicActionName ?associatedAtomicActionName \} \}}

Additionally, activation rules are specified to indicate when an atomic action has been pursued. Signals from the environment are processed and tested against parameters within activation rules. If such an activation rule is encountered, the ActivationTimestamp of this sensor's profile is updated to reflect the current time, $t_{n}$.

When sending signals from the environment, a Belief-rule Override Signal (BOS) may be specified as the value for a sensor parameter. The BOS is a cryptographic nonce that, when present, causes immediate activation of rule elements. If all of the sensor data parameters that are involved with the activation 
rule contain this BOS, then the rule will be ignored and the activation timestamp of the sensor will be immediately updated.

Activation rules and the ability to associate sensors with atomic actions increase the variety of sensors that may be placed within a home and incorporated into this approach. These sensors would generate signals that are sent to the $\mathrm{SH}$ agent and tested against activation rules to indicate performance of an atomic action. The properties of this ontology are presented in Table III.

TABLE III

THE PROPERTIES OF THE BELIEF-BASE ONTOLOGY

\begin{tabular}{ll}
\hline \hline \multicolumn{1}{c}{ Property } & \multicolumn{1}{c}{ Sensor Properties } \\
\hline $\begin{array}{l}\text { SensorID } \\
\text { SensorLabel }\end{array}$ & A unique ID for the sensor \\
AensorLocation & A human-friendly label \\
DateAdded & The date that the sensor record was added \\
AssociatedAtomic & $\begin{array}{l}\text { Used to link the sensor belief base/activation state to } \\
\text { atomic actions in the goal ontology/Agent desires, } \\
\text { ActionName }\end{array}$ \\
such as GetCup. \\
Parameter & $\begin{array}{l}\text { A sensor parameter that is observed, such as } \\
\text { beaconRSSI } \\
\text { The value for a specific parameter, such as -71 }\end{array}$ \\
\hline \hline
\end{tabular}

\section{Intentions}

In this goal-driven agent, we need to detect an inhabitant's intention toward goals. In order to model this, goal-activation states are considered. These activation states represent the inhabitant's attitude to these goals. These activation states are:

1) option - any goal that is present in the goal ontology/agent desires. This is the default state for inhabitant goals.

2) active - a goal that an IR mechanism has determined is being pursued. There is only one active goal at one time.

3) suspended - a previously active goal that has been suspended.

4) assist - an active goal that has been encountered is assist condition.

Goal operational states reflect an inhabitant's attitude toward these goals as determined by the atomic actions that an inhabitant performs. As such, detection of an inhabitant's performance of atomic actions is an essential element of recognizing an intended goal.

The devised IR process leverages the previously described belief and goal ontologies, in order to assign goal activation states. Once assigned, these goal activation states are used as a basis to provide assistance and model the completion state of a goal.

In this approach, goals are composed of direct atomic actions and, optionally, atomic actions that are inherited from subgoals. Thus, completion of these atomic actions and those inherited from subgoals can be used to indicate an inhabitant's intended goal, when liveliness of the actions is considered. To help illustrate this, the progression of atomic actions relative to goal pursuit is presented in Fig. 5.

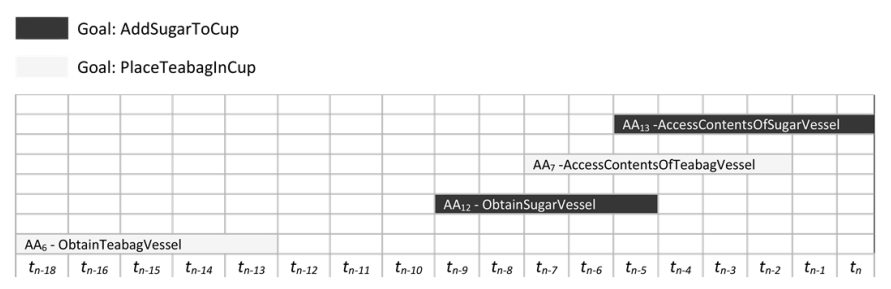

Fig. 5. The progression of atomic actions in relation to goals.

Given the window of activities presented in Fig. 5, we can determine the likely intended goal for a given time point by considering how recently atomic actions and related actions from a goal have been started. For example, at $t_{\mathrm{n}-20}$, no atomic actions were performed; therefore, we cannot determine any intended goal. Nevertheless, at $t_{\mathrm{n}-19}$, pursuit of an atomic action has started, as determined by a rule in the sensor belief base; therefore, from $t_{\mathrm{n}-19}$ until $t_{\mathrm{n}-11}$, we can ascertain that the intended goal is PlaceTeabagInCup. At $t_{\mathrm{n}-10}$, another action is performed belonging to the AddSugarToCup goal. As this is the most recent/lively activity, we can assume that from $t_{\mathrm{n}-10}$ until $t_{\mathrm{n}-8}$ this will be the intended goal. Similarly, from $t_{\mathrm{n}-8}$ until $t_{\mathrm{n}-6}$, we can assume that PlaceTeaBagInCup is the intended goal and then from $t_{\mathrm{n}-6}$ onwards we can assume that the AddSugarToCup is an intended goal. In this window of activity, is it possible to determine the intended goal after atomic actions have been performed. Additionally, when considering the goal hierarchy presented in Table II and Fig. 3, we can determine that the intended goals in Fig. 5 are subgoals of an overall goal; in this case, we can assume that an inhabitant is going to make some form of sweetened tea.

There can, however, be overlap of the performance of atomic actions, which can produce a scenario where for a given time point, the intended goal is not as clear. An example of such an activity progression is presented in Fig. 6.

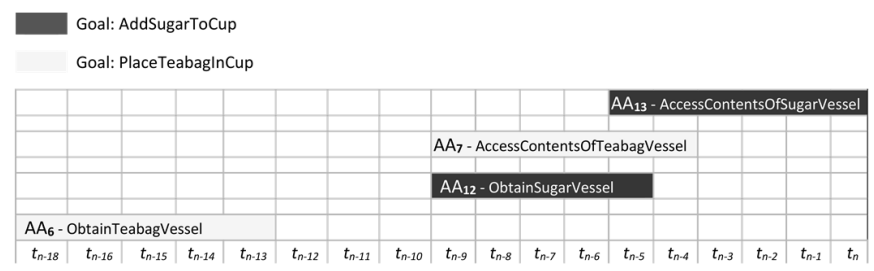

Fig. 6. The progression of overlapping atomic actions in relation to goals.

In Fig. 6, sole consideration of $t_{\mathrm{n}-10}$ makes it unclear which goal an inhabitant intends to pursue as there are two atomic actions simultaneously being detected. When considering previously detected atomic actions and the number of actions required to complete a goal, it is, however, possible to determine an intended goal. For example, at point $t_{\mathrm{n}-10}$, the inhabitant intended to pursue the PlaceTeabagInCup goal, because, at $t_{\mathrm{n}-19}$, a related atomic action was performed.

Using a combination of the time difference between $t_{\mathrm{n}}$ and any previously started atomic actions, it is possible to judge the liveliness of tasks. Combining liveliness of goals with the current completion state of that goal in an activity window will, in turn, allow determination of which goal is most likely being pursued. To further increase the accuracy of IR, weighting can be applied to goals where atomic actions that have an IsKeyAction property set to true have been started. Finally, weighting can be applied to goals that have historically set a trend for that time of day. 
An IR method encompassing these concepts has been conceived, adopting the following approach. All goals that have been modeled are considered and ranked by calculating the Mean Goal Liveliness (MGL) of these goals; these rankings are placed in the set $G_{x}$. This ranking considers how recently associated atomic actions have been initiated, $A_{i} t_{\Delta}$, for a given time point $t_{n}$ or the MGL value of subgoals, $S M V_{x}$, and the number of atomic actions and subgoals that are in each goal. Additionally, weighting is applied by adding 0.25 to $W_{k}$ for goals that have at least one key action performed and 0.20 to $W_{h}$ for goals that have historically been pursued in that time frame. Historical goal information was not recorded in this particular implementation, however, is present for future use. These weighting values were decided upon by iterative development and comparison of the performance of values ranging from 0.0 to 1.0 , which incremented in 0.05 steps. For the test goal detection, this offered good detection performance with a low weighting value. These factors are used to calculate the MGL, as shown in the equation presented in (2).

$$
\begin{gathered}
M G L_{G_{x}}=\left(A_{x}=\frac{1}{\left(n+W_{k}+W_{h}\right)} \sum_{i=1}^{n} A_{i} T_{\Delta}=\frac{1}{\left(n+W_{k}+W_{h}\right)}\right. \\
\left.=\left\{A_{i} T_{\Delta 1}, \ldots, A_{i} T_{\Delta i}\right\}\right) \\
+\left(S_{x}=\frac{1}{\left(n+W_{k}+W_{h}\right)} \sum_{i=1}^{n} S M V_{i}=\frac{1}{\left(n+W_{k}+W_{h}\right)}\right. \\
\left.=\left\{S M V_{1}, \ldots, S M V_{i}\right\}\right)
\end{gathered}
$$

Once the set $G S_{x}$ has been computed, the values within can be ranked with the aim of identifying an inhabitant's intended goal, as indicated by the lowest MGL value within the restrictions set by the goal recognition rules. These rules cover goal adoption, performance and recognition.

To model these goal recognition rules, Allen's temporal logic, a highly developed theory of actions, was considered and incorporated in the devised IR approach. The relationships between intervals modeled in Allen's temporal logic is shown in Table IV.

TABLE IV

THE RELATIONSHIP BETWEEN INTERVALS AS MODELED IN ALLEN'S TEMPORAL LOGIC

\begin{tabular}{ccc}
\hline \hline Relation & Symbol/Inverse Symbol & Pictorial example \\
\hline $\mathrm{X}$ before $\mathrm{Y}$ & $</>$ & $\mathrm{xxx}$ yyy \\
$\mathrm{X}$ equal $\mathrm{Y}$ & $=/$ & $\mathrm{xxx}$ \\
$\mathrm{X}$ meets $\mathrm{Y}$ & $\mathrm{m} / \mathrm{mi}$ & yyy \\
$\mathrm{xxxyyy}$ \\
$\mathrm{X}$ overlaps $\mathrm{Y}$ & $\mathrm{o} / \mathrm{oi}$ & $\mathrm{xxx}$ \\
& & yyy \\
$\mathrm{X}$ during $\mathrm{Y}$ & $\mathrm{d} / \mathrm{di}$ & xxx \\
& & yyyyyy \\
X starts $\mathrm{Y}$ & $\mathrm{s} / \mathrm{si}$ & xxx \\
& & yyyyy \\
$\mathrm{X}$ finishes $\mathrm{Y}$ & $\mathrm{f} / \mathrm{fi}$ & xxx \\
& &
\end{tabular}

This temporal logic is incorporated into the rules for goal pursuit, goal performance, subgoal relationships, performance of atomic actions, determination of goal activation states, and identification of the intended inhabitant goal. These rules are presented in Table V.

TABLE V

\begin{tabular}{|c|c|}
\hline Rule & Inherited Goals \\
\hline $\mathrm{G}=\{\mathrm{G} x ; \mathrm{x}=1, \ldots, \mathrm{N}\}$ & A set of goals $\left(G_{x}\right)$ that an inhabitant may pursue. \\
\hline \multirow{6}{*}{$\begin{array}{l}\mathrm{G}=<\mathrm{PC}, \mathrm{A}, \mathrm{MGL}, \mathrm{MPC} \\
\mathrm{AC}, \mathrm{SG}, \mathrm{AchC}>\end{array}$} & $\begin{array}{l}\text { Goals may have a range of associated properties, } \\
\text { including the following }\end{array}$ \\
\hline & $\begin{array}{l}\text { Preconditions }(P C) \text { they must meet to be } \\
\text { considered for pursuit. } \\
\text { Atomic Actions }(A) \text { that need to be } \\
\text { performed to complete the goal tasks. }\end{array}$ \\
\hline & $\begin{array}{l}\text { - } \quad \text { Mean Goal Liveliness values }(M G L) \text { as } \\
\text { covered previously. }\end{array}$ \\
\hline & $\begin{array}{l}\text { - Assist Conditions }(A C) \text { to indicate if a goal is } \\
\text { taking too long to pursue, and hence the } \\
\text { inhabitant needs assistance. }\end{array}$ \\
\hline & $\begin{array}{l}\text { - Subgoals }(S G) \text { that are needed to complete } \\
\text { the goal, }\end{array}$ \\
\hline & $\begin{array}{l}\text { Achievement Conditions }(A c h C) \text { indicate the } \\
\text { circumstance where a goal is achieved, }\end{array}$ \\
\hline
\end{tabular}

THE RULES FOR GOAL PURSUIT, PERFORMANCE OF SUBGOALS, PERFORMANCE OF ATOMIC ACTIONS, DETERMINATION OF GOAL ACTIVATION STATES AND IDENTIFICATION OF THE INTENDED INHABITANT GOAL.

$G_{x}\{o, d, m\} G_{y}$ $\mathrm{SG}_{\mathrm{y}}\{\mathrm{o}, \mathrm{d}, \mathrm{m}\} \mathrm{G}_{\mathrm{x}}$ $\mathrm{G}_{y}\{\mathrm{o}, \mathrm{d}, \mathrm{m}\} \mathrm{SG}_{\mathrm{x}}$ $\mathrm{SG}_{\mathrm{y}}\{\mathrm{o}, \mathrm{d}, \mathrm{m}\} \mathrm{SG}_{\mathrm{x}}$ $\mathrm{G}_{\mathrm{x}} \mathrm{A}_{\mathrm{i}}\{\mathrm{o}, \mathrm{d}, \mathrm{m}\} \mathrm{G}_{\mathrm{y}} \mathrm{A}_{\mathrm{j}}$ $\mathrm{SG}_{\mathrm{x}} \mathrm{A}_{\mathrm{i}}\{\mathrm{o}, \mathrm{d}, \mathrm{m}\} \mathrm{G}_{\mathrm{y}} \mathrm{A}_{\mathrm{j}}$ $G_{x} A_{i}\{o, d, m\} S G_{y} A_{j}$ $S_{\mathrm{x}} A_{\mathrm{i}}\{o, d, m\} \mathrm{SG}_{\mathrm{y}} \mathrm{A}_{\mathrm{j}}$ $\mathrm{G}_{\mathrm{x}} \mathrm{A}_{\mathrm{i}} \wedge \mathrm{SG}_{\mathrm{y}} \mathrm{A}_{\mathrm{j}}\{\mathrm{f}\} \mathrm{FG}_{\mathrm{x}}$

The performance of any goals/subgoals may overlap, meet or occur simultaneously.

The performance of any atomic actions may overlap, meet or occur simultaneously.

Goals may be completed when all non-optional atomic actions are complete.

$\left\{U S G_{x} \subseteq U S G ; x\right.$

$\in U S G_{x} \Leftrightarrow x=\left|P C_{G_{x}}\right|$ Goals that have no preconditions are placed in the $>0\}$

$\left\{P C G S_{x} \subseteq P C G S ; x\right.$ unrestricted goal set, $U G S_{x}$

Goals, which have preconditions, which have been met, are placed in the preconditions-met goal set, $\left.\in P C G S_{x} \Leftrightarrow x=P C_{G_{x}}\right\} \quad P C G S_{x}$.

The set containing the computed MGL values for goals, $G S_{x}$, is reduced to restrict the candidate

$\mathrm{CGSx}=\{\mathrm{x} \in$ goals to those that have met heir preconditions,

$\left(U G S_{x} \cap P C G S_{x} \cap G S_{x}\right) ; \quad$ Poals to those that have met goals with no preconditions $U G S_{x}$ and $\left.A \operatorname{ch} G_{x}\right\}$ excludes achieved goals $(A c h G)$. This forms the set $C G S_{x}$. This represents goals in the option state.

$$
\begin{aligned}
& \left\{\operatorname{Sus}_{x} \subseteq \operatorname{SusG} ; x\right. \\
& \in \operatorname{Sus} G_{x} \Leftrightarrow x \\
& \left.=\operatorname{Sus}_{C G S_{x}}\right\}
\end{aligned}
$$

A suspended Goal set (SusG) consists of goals from the set $C G S$ that have encountered their Suspend Condition $(\operatorname{SusC})$. This represents a goal that is in the suspend state.

$I G=\{x \in \mathrm{CGSx} \Leftrightarrow x$ The currently Intended Goal $(I G)$ is one that has $=\min \left\{M G L_{C G S_{x}}\right\} ; x$ the lowest MGL in the set candidate goal set $C G S$ $\left.\notin \operatorname{Sus} G_{x}\right\}$ and is not in the $\operatorname{Sus} G$ set. This represents the goal in the active state.

$A G=\left\{I G \Leftrightarrow A C_{I G}\right\} \quad$ An Assist Goal $(A G)$ is a currently intended goal that has encountered its Assist Condition. An Achieved Goal is an $I G$, which has encountered its achievement condition. On $\mathrm{AchG}=\left\{I G \Leftrightarrow A \operatorname{ch} C_{I G}\right\} \quad$ achievement, the parameters that calculate the MGL values are reset to zero removing these goals from consideration.

\section{Evaluation}

\section{A. Experimental design}

To evaluate this approach to IR, an implementation was produced through an evaluation platform called INSigHt 
(Intelligent ageNt Smart Home). This platform is a custom BDI agent architecture leveraging semantic web technologies through the ontological components previously outlined.

INSigHt was implemented as a web service-based system, specifically using Java servlets hosted on Tomcat servers. These servlets hosted an implementation of the BDI agent architecture and IR mechanism, previously outlined. In this implementation, Virtuoso was used as the ontological database. Semantic reasoning on this ontological database was facilitated though JENA and Pellet. A relational database was used to log activity and store metadata.

This architecture splits components into two classes, those based within the $\mathrm{SH}$ and those resigning on a server. These components communicate using REST and JSON.

Beliefs about activities in the environment are generated from a simple set of sensors affixed to objects in a SH. These sensors may vary, however, in this instance, inexpensive Bluetooth Low Energy (BLE) beacons were used. In this particular instance, Estimote sticker beacons were selected. Beacons are affixed to objects that an inhabitant may interact with during performance of their goals. Beacons are monitored by an application running on an inhabitant smart device such as a smart phone or smartwatch in order to provide data about inhabitant-object proximity, temperature, and accelerometer information. The smart device relays this information to a web service, which in turn updates the assistive agent's belief base ontology.

Thermal and accelerometer information is broadcasted at $10 \mathrm{~Hz}$. Inhabitant-object proximity information can be used to provide context. Context can be optionally leveraged by goal preconditions to reduce the search space for goals that an inhabitant is likely to pursue, increasing recognition accuracy.

Additionally, a unique inhabitant ID and the push messaging ID for that device is sent to the belief endpoint. This devicebased push messaging addresses each user goal assistance. The belief ontology is a specific per inhabitant instance incorporating an inhabitant ID. These are extended from a common base belief ontology, extending goal and belief reusability. The push messaging ID and associated information is stored in a relational database alongside inhabitant IDs. This information is provided automatically on the first use of the application.

Finally, due to the separation of the belief base from activity models, other sensor types may be used to update this belief base by providing activation rules within the sensor ontology and a listener within the environment. During prototyping of this system, contact sensors were used in place of BLE beacons.

Desires in this approach represent the goals that an inhabitant may perform. These are modeled from domain knowledge and are stored in goal ontology. Once goals are modeled, their atomic actions are linked to sensor activity by the AssociatedAtomicActionName property of the sensor class in the belief ontology.

When desires are modeled and beliefs are observed, the IR rules, detailed earlier, can operate with this information to determine the classification of goals as achieved, assisted or intended. These goal-recognition rules are enacted through the SPARQL rules previously shown and a Java-based implementation of the formalism presented.
These rules are used to process the beliefs of the agent on a $500 \mathrm{~ms}$ interval to classify goals and determine the need to offer assistance. This interval was chosen to provide a balance of response speed, resource consumption, and to provide greater scalability.

The implementation of this platform is presented in Fig. 7.

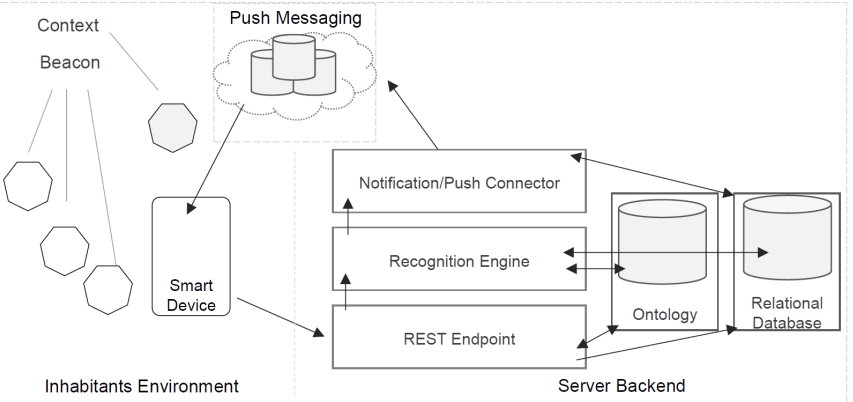

Fig. 7. An illustration of the architecture of the implemented evaluation platform; to reduce complexity the web-based user interface components used to set sensor associations and goals are not shown.

The recognition engine currently provides assistance by providing a list of steps required to achieve a goal and sending them to the inhabitant smart device via push messaging. Push messaging allows rapid updating of mobile clients in a manner that conserves battery on the mobile device. Once the smart device receives a message containing the list of steps, it alerts the inhabitant and presents the remaining steps required.

\section{B. Data collection and test procedure}

To collect data as an evaluation testbed for our approach, smart devices and devices and a web interface were used in three evaluation scenarios. These are:

1) web-based simulation where sensor interactions were simulated through a Web user interface (UI). This allows evaluation of the IR component and agent without sensor errors becoming a factor.

2) real-word simulation within a $\mathrm{SH}$ where a smart device was placed in a test user's pocket. This allows evaluation of the system in the real world, with beacons being read from the pocket of inhabitants. The smart device was placed in a pocket nearest the dominant hand of the user.

3) real-world simulation within a $\mathrm{SH}$ with the smart device affixed to the forearm of a test user. This simulates use of a smart watch to monitor user/environment interaction. At the time of evaluation, no standalone smart watch could operate with the beacon sensors used in this evaluation and so their use was emulated. The mobile phone app and beacons are presented in Fig. 8.

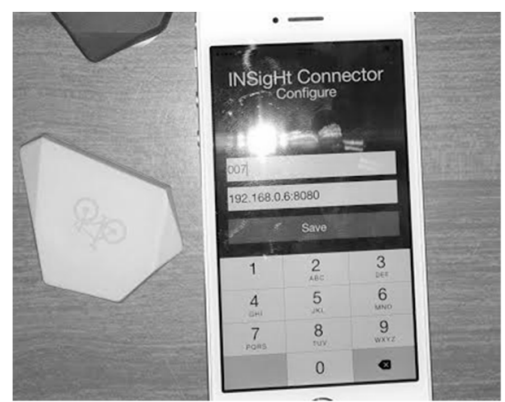

Fig. 8. The mobile phone and beacons used in real-world simulation. 
During this evaluation process, a ground truth record of activity performance was produced by manual annotation. This ground truth was primarily recorded by the inhabitant of the $\mathrm{SH}$ that was performing the activities. These manual annotations noted the time and date of the activity, the target activity, and the order of objects interacted with.

During the evaluation process, six activities were performed. These were variations of making coffee and making tea. These activities were chosen as they share common subgoals and may represent variations of a task. As such, they would have the capability to confuse the IR mechanism and can highlight the scalability of this reusable model.

A statistical power test was used to determine the number of samples required to produce a power of 0.90 within a margin of 0.05 , a Type I error rate of $5 \%$, and a standard deviation of 0.10 . This calculation showed that for results to express this statistical power, a minimum of 44 samples/iterations were required.

These activities were used to evaluate performance through 253 activity iterations. 120 iterations were performed in evaluation scenario 1 over two days. 85 iterations were performed in evaluation scenario 2 and 48 iterations were performed in evaluation scenario 3 . The evaluation process in scenarios 2 and 3 involved natural activity performance within a test user's real home across a period of three months.

The Received Signal Strength Indication (RSSI) parameter was used to determine the distance between the sensor and the smart device. To facilitate a more flexible real world evaluation, the smart device sent the BOS in place of the RSSI sensor parameter, under certain circumstances. The use of the BOS allowed dynamic reconfiguration of the evaluation scenario by changing the activation profile to one relevant for each use case, scenario 2 or 3 . If this override were not sent, the belief base would have to be modified frequently during testing to facilitate the particular scenario being evaluated.

Specifically, the in-app activation profiles would send the BOS when their specific RSSI threshold was encountered. These threshold values used were -80 decibel-milliwatts $(\mathrm{dBm})$ in scenario 1 and $-96 \mathrm{dBm}$ in scenario 2 . These values were selected through evaluation of the RSSI when objects affixed with beacons were being interacted with during test cases for these scenarios. Beacon accelerometer data is read from the beacons and sent to the backend, which are then processed against the activation rules to determine if activation occurred.

During evaluation, activities were performed with intended goals being identified by the assistive agent. Intended goals were then abandoned at random intervals, emulating forgetfulness. These abandoned, intended goals where then eligible to reach their assist condition. Once this assist condition was encountered, the goal's profile was sent to the Web UI and mobile device. This goal profile lists the goal name, actions that have been completed, and actions that need to be completed. This is the same goal profile format used by the assistance provisioning component of this project, which has been previously evaluated [29], [30].

In this specific evaluation, historical records were not used, although accounted for within the MGL calculation.

\section{Results}

To determine if each evaluation deviated from the ground truth in a statistically significant manner, a chi ${ }^{2}$ test was performed. $\mathrm{Chi}^{2}$ values were computed for each type of evaluation. These are compared to the critical $\mathrm{chi}^{2}$ value (chi ${ }^{2}$ crit) for a p-value of 0.05 . In this instance, $\mathrm{chi}^{2}{ }^{2}$ rit is 11.07 . If the $\mathrm{chi}^{2}$ for an evaluation is larger than $\mathrm{chi}^{2}$ crit, that evaluation has deviated from the ground truth in a statistically significant manner. The accuracy of this process is presented in Table VI and is followed by a discussion.

TABLE VI

GOAL RECOGNITION ACCURACY IN THE DEVISED IR-BASED AGENT

\begin{tabular}{cccc}
\hline \hline $\begin{array}{c}\text { Evaluation scenario } \\
\text { (Iterations) }\end{array}$ & Inhabitant goal (Iterations) & Accuracy & chi $^{2}$ \\
\hline & Making Black Coffee (20) & $100 \%$ & \\
Meb-based & Making White Coffee (20) & $100 \%$ & \\
simulation (120) & Making Green Tea (20) & $100 \%$ & 0 \\
& Making White Tea (20) & $100 \%$ & \\
& Making White Sugary Tea (20) & $100 \%$ & \\
Real-word & Making Black Coffee (15) & $66.67 \%$ & \\
simulation with a & Making White Coffee (15) & $60 \%$ & \\
smart device placed & Making Green Tea (20) & $70 \%$ & 10.76 \\
in the pocket (85) & Making White Tea (15) & $60 \%$ & \\
& Making White Sugary Tea (10) & $70 \%$ & \\
Real-world & Making Black Coffee (8) & $87.5 \%$ & \\
simulation with the & Making White Coffee (8) & $87.5 \%$ & \\
smart device being & Making Sugary White Coffee (8) & $75 \%$ & \multirow{2}{*}{ (1.5 } \\
affixed to the & Making Green Tea (8) & $87.5 \%$ & \\
forearm (48) & Making White Tea (8) & $87.5 \%$ & \\
\hline \hline
\end{tabular}

In the evaluation, a high degree of accuracy was achieved in goal recognition across two of the evaluation scenarios. Specifically, $100 \%$ accuracy was achieved in scenario 1 with $83.3 \%$ accuracy in scenario 2 . In the third scenario, acceptable levels of accuracy were achieved, $64.4 \%$. The results show statistical significance as shown through chi $^{2}$ tests.

In scenario 1, 100\% accuracy was achieved. This is because of the removal of errors introduced by the sensor component and possibly because of a more focused, single-minded, and direct activation procedure. This simulation exclusively tested the IR component and showed the capability of the devised formalism and the implemented agent.

Scenario 2 revealed this approach to perform most poorly. This was because of the low RSSI value of the testing profile. This low RSSI value expanded the physical consideration range of beacons. This caused a specific recurring issue related to object proximity confusion. An instance of this was coffee and tea making activities being confused as these vary by one sensor activation in the modeled goals, that is, the vessels for storing tea and coffee. Specifically, these vessels were placed beside one another in a cupboard. On movement of one vessel, others would be agitated and register movement within the allowed proximity range. This movement caused a race condition where the first sensor signal received was passed to the IR mechanism.

This error could be compensated for through a number of methods, such as using activation rules in the belief base, adding a signal filter in the smart phone application, using additional beacons or rearranging the environment to reduce confusion. Activation rules could be used to specify minimum movement levels for activation to be considered. Alternatively, rules incorporating tri-axial angle ranges could be modeled. 
Either of these rule-based approaches could be used to detect the intended interaction more robustly, as opposed to unintended agitation. Alternatively, a context beacon could be used to more accurately determine a beacon that is being interacted with, through trilateration. This, however, would require a per-room belief base reducing the reusability of such an approach.

A signal filter could be added to the smart phone application to ignore beacons generating minor movements when in proximity to beacons that are recording larger movements

Finally, a simple solution is possible through moving the relevant confusing vessels to easily discernable locations; in this particular test scenario, using different shelves to store each vessel would have remedied this issue.

Scenario 3 represents the most accurate use of this system in the real world and operated to a high degree of accuracy.

This system was tested under the class of Intended IR as the observed entity was aware of this operation. Ideally, this system would be tested under the Keyhole class of IR operation, as those suffering age-related illness may not be consistently aware that they are being monitored by such an assistive system. This highlights the need to deploy and evaluate the use of this system in an environment occupied by those with mild cognitive impairment. Through such an evaluation, the operation of this approach could be more thoroughly tested and improved.

This approach addresses some of the issues of current SHs, namely those related to inhabitant privacy, scalability, flexible activity variation and reusability.

Privacy is preserved by virtue of this system not requiring a record of the previous activity performance in order to operate. This approach is opposite to a number of contemporary assistive SHs, particularly those based on machine learning.

Improved scalability and adoption is provided using an IR mechanism that focuses on goal actions that are linked to individual sensors. This allows functionality to be realized through object-affixed beacon sensors that are relatively inexpensive and easily deployed in an environment.

Additionally, this system is intended to be cloud hosted with clients by simply deploying beacons and installing an application to their chosen smart device.

Finally, the implemented approach required low amounts of computational resources to operate. The evaluated system was able to reason about an inhabitant's intended goal typically within $140 \mathrm{~ms}$ and $160 \mathrm{~ms}$. The system was hosted on a device containing a $1.7 \mathrm{GHz}$ Intel Core 17 mobile CPU. The majority of this reasoning time was because of network latency from interacting with the relational and ontological databases.

The low CPU usage of this approach in combination with its cloud-based nature leads to a technically scalable approach that can assist multiple residents through use of user profiles.

Activities are modeled through inhabitant goals, which flexibly model activities based on ontological concepts and inheritance of atomic actions. These activity models are defined through a simple web interface, removing the requirement for specialist knowledge beyond that of the activity. In the specific example given earlier in this article, presented in Fig. 2, 16 goals are modeled through a combination of seven goals that contain associated atomic actions.
Reusability of this system is an improvement upon previous approaches through modeling concepts using ontological techniques, use of a logic driven approach with the ability to operate without previous activity records.

The previously suggested improvements are being planned and are discussed in the further study Section.

\section{V.FURTHER STUDY}

Future study is separated into three tasks; further evaluation and improvement, integration into a complete system, and evaluating the possibility of making this system available to the research community.

Further evaluation is required to focus on using this system in a Keyhole manner to ensure that the approach operates with users who are suffering from age-related mental impairments. Moreover, as the evaluated ADLs were of moderate complexity, a more complex range of ADLs should be modeled and evaluated. Additionally, evaluation would be required to test functionality with multiple occupants.

Finally, modifications should be made to the evaluation approach to incorporate advanced sensor activation rules to better prevent wrongly detected activity performance. This system will be incorporated in a future research project; this project should provide opportunity for some of this extended evaluation.

Once the approach and system has been evaluated and improved, efforts should be made to integrate the companion, assistance-provisioning, components produced within [29], [30].

These companion components leverage the ontological nature of this system to reuse/share data and leverage automated reasoning through semantic rules such as SPARQL and SWRL. Specifically, these components process video files and identify actions depicted within. These identified actions, and their semantically compatible variations, are subsequently placed in a media annotation ontology. This media annotation ontology is incorporated into a planning component, which uses the goal ontology to match guiding video files to goals whose assistance condition has been encountered.

Integration would deliver a complete solution. Currently, integration is loose, although manual messages pass to and from each component's REST endpoint. This facilitates dynamic testing and iterative improvement of each component.

To make this system widely accessible to the research community there are two main avenues that will be investigated; open sourcing the current codebase and providing an implementation through software as a service.

\section{CONCLUSION}

This article presents an Intelligent Agent architecture and IR mechanism that may be used to form an AAL system to assist with ADLs within a SH. Specifically, the approach can be used to enable an $\mathrm{SH}$ environment by affixing sensors to objects within a home that will be interacted with during the course of the performance of ADLs.

To the best of the authors' knowledge, this is the first time that an IR-based Intelligent Agent platform that models inhabitant goals has been successfully devised for, and applied to, this problem domain. 
This approach addresses some issues SHs have exhibited related to privacy, reusability, applicability, and scalability.

Privacy is addressed in two ways. This approach does not rely on a data-driven approach to provide ADL recognition where a large number of sensor records are captured throughout the daily life of the inhabitant and learned.

Sensors incorporated are simple object sensors whose interaction indicates performance of an atomic action when certain activation conditions are encountered. A large sensor suite producing sensitive data such as recorded video, recorded audio or occupant-tracking records is not required for this approach to function.

Reusability is catered for in a number of ways, prominently through the use of ontologies and modeling activities as inheritable goals. Ontologies facilitate sharing and linking of data amongst many concepts and components. This approach functions with companion-assistive components that reuse modeled goals to perform analysis on video files. This video analysis identifies depicted tasks within video files and provides assistance through dynamic planning.

This approach is applicable to a wide number of environments through formal separation of sensors from atomic actions and integration of a cloud-based backend. Separation of sensor modeling through the use of a BDI architecture allows sensors to be related to atomic actions independent of activity structures. This separation allows goals to be reused across a number of SHs where sensors are simply associated with atomic actions and are given a SensorLocation property per environment, reducing the effort needed to model data for each $\mathrm{SH}$ and increasing applicability.

Additionally, the devised approach is cloud based, facilitating integration of new SHs by placing sensors and a listener in an internet-accessible home; with no local computation infrastructure.

Scalability is addressed through use of inheritable goal structures and focusing on the proven scalability of objectsensor interactions in recognizing a range of ADLs modeled as plan-like structures [19]. As the evaluation shows, modeled goals may be reused to form components of other goals. This facilitates scalability of modeled activities by reusing defined components to produce new goals, which in turn may have their own activation and assistance conditions. Additionally, these goal models are not tied to a specific environment and may be reused within each environment.

As shown in the presented evaluation, this first-generation Intelligent Agent and IR SH system for AAL performed well, with minimal issues appearing in this early stage evaluation.

Issues that were made apparent were related to object interaction where beacon placement in the real world caused object confusion and subsequent errors, that is, when objects were placed in extreme proximity. A number of methods to cater for these issues have been proposed by the authors; these should be integrated in future with a subsequent evaluation. In a web-based simulation, this approach performed without any such issues.

Further study incorporating improvements with subsequent evaluations have been proposed. Additionally, the authors intend to make this system available to the research community and partners through open sourcing the project or providing it through software as a service, if possible.

\section{ACKNOWLEDGMENT}

The authors would like to thank the Northern Ireland Department of Education and Learning for partial support of this research.

\section{REFERENCES}

[1] United Nations, World Population Ageing 2009 (Population Studies Series), Pap/Cdr Ed. 2010.

[2] d'Alessandro E. De Luca, S. Bonacci, and G. Giraldi, "Aging populations: the health and quality of life of the elderly.," Clin. Ter., vol. 162 , no. 1, p. e13, 2011.

[3] D. J. Cook and S. K. Das, "How smart are our environments? An updated look at the state of the art," Pervasive Mob. Comput., vol. 3, no. 2 , pp. 53-73, Mar. 2007.

[4] M. Chan, D. Estève, C. Escriba, and E. Campo, "A review of smart homes- present state and future challenges.," Comput. Methods Programs Biomed., vol. 91, no. 1, pp. 55-81, Jul. 2008.

[5] L. Chen, J. Hoey, C. D. Nugent, D. J. Cook, and Z. Yu, "SensorBased Activity Recognition," IEEE Trans. Syst. Man, Cybern. Part C (Applications Rev., pp. 1-19, 2012.

[6] M. P. Poland, C. D. Nugent, H. Wang, and L. Chen, "Smart Home Research:Projects and Issues," Int. J. Ambient Comput. Intell., vol. 1, no. 4, pp. 32-45, Jan. 2009.

[7] L. Bao and S. Intille, "Activity recognition from user-annotated acceleration data," Pervasive Comput., pp. 1-17, 2004.

[8] E. Tapia, S. S. Intille, and K. Larson, "Activity Recognition in the Home Using Simple and Ubiquitous Sensors Pervasive Computing," in Pervasive Computing, vol. 3001, A. Ferscha and F. Mattern, Eds. Berlin, Heidelberg: Springer Berlin / Heidelberg, 2004, pp. 158175.

[9] D. J. Cook and M. Schmitter-Edgecombe, "Assessing the quality of activities in a smart environment.," Methods Inf. Med., vol. 48, no. 5, pp. 480-485, 2009.

[10] T. van Kasteren and B. Krose, "Bayesian activity recognition in residence for elders," 3rd IET Int. Conf. Intell. Environ. (IE 07), vol. 2007, pp. 209-212, 2007.

[11] U. Maurer, A. Rowe, A. Smailagic, and D. Siewiorek, "Location and activity recognition using ewatch: a wearable sensor platform," in Ambient Intelligence in Everyday Life, Y. Cai and J. Abascal, Eds. Berlin, Heidelberg: Springer-Verlag, 2006, pp. 86-102.

[12] N. Ravi, N. Dandekar, P. Mysore, and M. L. Littman, "Activity recognition from accelerometer data," in Proceedings of the 17th conference on Innovative applications of artificial intelligence Volume 3, 2005, pp. 1541-1546.

[13] M. Stikic and B. Schiele, "Activity Recognition from Sparsely Labeled Data Using Multi-Instance Learning," in Location and Context Awareness, 2009, pp. 156-173.

[14] D. L. Vail, M. M. Veloso, and J. D. Lafferty, "Conditional Random Fields for Activity Recognition," in Proceedings of the 6th International Joint Conference on Autonomous Agents and Multiagent Systems, 2007, pp. 235:1-235:8.

[15] C. Sutton, A. McCallum, and K. Rohanimanesh, "Dynamic Conditional Random Fields: Factorized Probabilistic Models for Labeling and Segmenting Sequence Data," J. Mach. Learn. Res., vol. 8, pp. 693-723, May 2007.

[16] O. Brdiczka, J. L. Crowley, and P. Reignier, "Learning situation models in a smart home.," IEEE Trans. Syst. Man. Cybern. B. Cybern., vol. 39, no. 1, pp. 56-63, Feb. 2009.

[17] L. Chen and C. Nugent, "A logical framework for behaviour reasoning and assistance in a smart home," Int. J. Assist. Robot. Mechatronics, pp. 20-34, 2008.

[18] B. Bouchard, S. Giroux, and A. Bouzouane, "A smart home agent for plan recognition," Adv. Artif. Intell., vol. 1, no. 5, pp. 53-62, 2006.

[19] L. Chen, C. D. Nugent, and H. Wang, "A Knowledge-Driven Approach to Activity Recognition in Smart Homes," IEEE Trans. Knowl. Data Eng., vol. 24, no. 6, pp. 961-974, Jun. 2012.

[20] N. Yamada, K. Sakamoto, and G. Kunito, “Applying ontology and probabilistic model to human activity recognition from surrounding things," IPSJ Digit. Cour., vol. 3, pp. 506-517, 2007.

[21] V. Vassilev, M. Ulman, and K. Ouazzane, "ONTOCARER: AN ONTOLOGICAL FRAMEWORK FOR ASSISTIVE AGENTS 
FOR THE DISABLED," in The Third International Conference on Digital Information Processing and Communications (ICDIPC2013), 2013, pp. 404-416.

[22] F. Latfi, "Ontology-based management of the telehealth smart home, dedicated to elderly in loss of cognitive autonomy," CEUR Workshop Proc., 2007.

[23] M. Klein, A. Schmidt, and R. Lauer, "Ontology-centred design of an ambient middleware for assisted living: The case of soprano," 30th Annu. Ger. Conf. Artif. Intell., 2007.

[24] R. Hervás, J. Bravo, J. Fontecha, and V. Villarreal, “Achieving Adaptive Augmented Reality through Ontological ContextAwareness applied to AAL Scenarios," J. Univers. Comput. Sci., vol. 19, no. 9, pp. 1334-1349, 2013.

[25] B. Chandrasekaran, J. R. Josephson, and V. R. Benjamins, "What are ontologies, and why do we need them?," IEEE Intell. Syst., vol. 14, no. 1, pp. 20-26, Jan. 1999.

[26] B. Das, N. C. Krishnan, and D. J. Cook, "Automated Activity Interventions to Assist with Activities of Daily Living," Agents Ambient Intell. Achiev. Challenges Intersect. Agent Technol. Ambient Intell., vol. 12, pp. 137-159, 2000.

[27] P. Rashidi and A. Mihailidis, "A survey on ambient-assisted living tools for older adults.," IEEE J. Biomed. Heal. informatics, vol. 17, no. 3, pp. 579-90, May 2013.

[28] J. Rafferty, L. Chen, C. Nugent, and J. Liu, "Goal Lifecycles and Ontological Models for Intention Based Assistive Living within Smart Environments," Comput. Syst. Sci. Eng., vol. 30, no. 1, pp. 7 18,2015

[29] J. Rafferty, C. Nugent, J. Liu, and L. Chen, "Automatic Metadata Generation Through Analysis of Narration Within Instructional Videos," J. Med. Syst., vol. 39, no. 9, pp. 1-7, 2015.

[30] J. Rafferty, C. Nugent, J. Liu, and L. Chen, "A mechanism for nominating video clips to provide assistance for instrumental activities of daily living," in Ambient Assisted Living and Daily Activities, vol. 9455, Puerto Varas: Springer, 2015, pp. 65-76. M. Wooldridge and N. R. Jennings, "Intelligent agents: theory and practice," Knowl. Eng. Rev., vol. 10, no. 02, p. 115, Jul. 2009. F. Sadri, "Ambient intelligence," ACM Comput. Surv., vol. 43, no. 4, pp. 1-66, Oct. 2011.

[33] N. Jennings, "An agent-based approach for building complex software systems," Commun. ACM, vol. 44, no. 4, pp. 35-41, 2001. M. Ball and V. Callaghan, "Managing Control, Convenience and Autonomy," J. Ambient Intell. Smart Environ., 2012.

J. Thangarajah, J. Harland, and N. Yorke-Smith, "A soft COP model for goal deliberation in a BDI agent," Proc. CP, 2007.

[36] A. Rao and M. Georgeff, "Modeling rational agents within a BDIarchitecture," Readings in agents, 1997.

[37] F. Bellifemine, A. Poggi, and G. Rimassa, "Developing multi-agent systems with JADE," Intell. Agents VII Agent Theor. ..., 2001.

[38] N. Howden and R. Rönnquist, "JACK intelligent agents-summary of an agent infrastructure," 5th Int. Conf. Auton. agents, 2001. A. Pokahr, L. Braubach, and W. Lamersdorf, "Jadex: A BDI reasoning engine," Multi-Agent Program., 2005.

[40] A. Bawa, S. Bhatia, and V. K. Attri, "A REVIEW ON AGENT ORIENTED SOFTWARE ENGINEERING," Int. J. Adv. Res. Comput. Commun. Eng., vol. 4, no. 4, pp. 421-423, 2015.

[41] S. Arora, C. P. Agrawal, P. Sasikala, and A. Sharma,

"Developmental approaches for Agent Oriented system - A critical review," 2012 CSI Sixth Int. Conf. Softw. Eng., pp. 1-5, Sep. 2012.

[42] A. Bleda, R. Maestre, A. Jara, and A. Skarmeta, "Ambient Assisted Living Tools for a Sustanaible Aging Society," in Resource Management in Mobile Computing Environments, vol. 3, Springer International Publishing, 2014, pp. 193-220.

[43] J. Allen, H. Kautz, R. Pelavin, and J. Tennenberg, "A formal theory of plan recognition and its implementation," Reason. About Plans, pp. 1-67, 1991

[44] P. R. Cohen, C. R. Perrault, and J. F. Allen, "Beyond QuestionAnswering," in Stmtegies for Natural Language Processing. Lawrence Erlbaum, Englewood Cliffs, 1981.

[45] C. W. Geib and R. P. Goldman, "Partial Observability and Probabilistic Plan/Goal Recognition," IJCAI-05 Work. Model. Others from Obs., p. 6, 2005.

[46] B. Bouchard, S. Giroux, and A. Bouzouane, "A keyhole plan recognition model for Alzheimer's patients: first results," Appl. Artif. Intell., vol. 22, no. July, pp. 1-34, 2007.

[47] F. Sadri, "Logic-based approaches to intention recognition," Handb.
Res. Ambient Intell. Smart Environ. Trends Perspect., 2010.

[48] K. Oyama, C. Chang, and S. Mitra, "Inference of Human Intentions in Context Aware Systems," in Inference of Human Intentions in Context Aware Systems, 2011, pp. 2011-2013.

[49] M. Huber, "JAM: A BDI-theoretic mobile agent architecture," Proc. third Annu. Conf. Auton. Agents, pp. 236-243, 1999.

[50] F. Sadri, "Intention Recognition with Event Calculus Graphs," 2010 IEEE/WIC/ACM Int. Conf. Web Intell. Intell. Agent Technol., pp. 386-391, Aug. 2010.

[51] F. Krüger, M. Nyolt, K. Yordanova, A. Hein, and T. Kirste, "Computational state space models for activity and intention recognition. A feasibility study,” PLoS One, vol. 9, no. 11, 2014.

[52] K. Yordanova and T. Kirste, “A Process for Systematic Development of Symbolic Models for Activity Recognition," $A C M$ Trans. Interact. Intell. Syst., vol. 5, no. 4, pp. 20:1-20:35, Dec. 2015 .

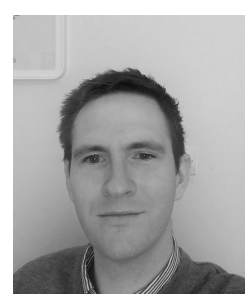

Joseph Rafferty (M'14) received the B.Eng. degree in Computer Science from Queens University Belfast, the M.Sc. degree in Computing from Ulster University and the $\mathrm{PhD}$ degree in Computer Science from Ulster University. He is currently a Research Fellow at the School of computing, Ulster University. His research interests include intention recognition, smart environments, agent based systems, connected health, sensor technology, planning and intelligent systems.

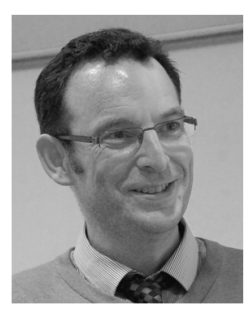

Chris D. Nugent (M'96) received the B.Eng. degree in electronic systems and the D.Phil. degree in biomedical engineering both from the University of Ulster, U.K. He is currently a Professor of biomedical engineering with the School of Computing and Mathematics, Ulster University. His research addresses intelligent data analysis for Smart Environments and the design and evaluation of pervasive and mobile solutions within the context of ambient assisted living.

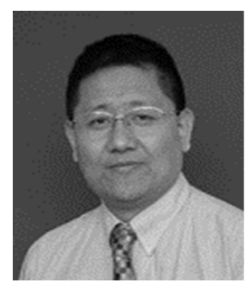

Jun Liu received the BSc and MSc degrees in applied mathematics, and the $\mathrm{PhD}$ degree in information engineering from Southwest Jiaotong University, Chengdu, China, in 1993, 1996, and 1999, respectively. $\mathrm{He}$ is currently a Senior Lecturer in Computer Science at Ulster University, Northern Ireland, United Kingdom. He has been working in the field of AI for many years. His current research interests include logic and reasoning methods for intelligent systems and formal verification; intelligent DSSs and information management, with applications in health care, engineering, and industry field, etc. (e.g., safety and risk analysis; situation awareness and emergency systems; scenario/activity recognition); information fusion and data combinations; data mining and KBS; applied computational intelligence for uncertainty analysis and optimization. He is a member of the IEEE.

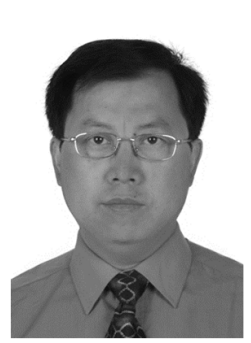

Liming Chen is Professor of Computer Science at the School of Computer Science and Informatics, De Montfort University, UK. He received his B.Sc. and M.Eng. from Beijing Institute of Technology, China, and a Ph.D. degree in Artificial Intelligence from De Montfort University, UK. His current research interests include activity recognition, intelligent systems, smart environment and assisted living. He has published over 160 research papers and currently the coordinator of the EU H2020 MSCA ACROSSING project. 